\title{
Análise de risco sócio-ambiental para comprometimento pleural na pneumonia grave em crianças menores de 5 anos
}

\author{
Karla Danielle B. P. Costa Pinto, ${ }^{1}$ Ruben R. Schindler Maggi ${ }^{1}$ \\ e João Guilherme B. Alves ${ }^{2}$
}

Como citar Pinto KDBPC, Maggi RRS, Alves JGB. Análise de risco socio-ambiental para comprometimento pleural na pneumonia grave em crianças menores de 5 anos. Rev Panam Salud Publica. 2004;15(2):104-9.

RESUMO Objetivo. Determinar fatores de risco sócio-ambientais associados ao desenvolvimento de comprometimento pleural em crianças de 3 a 59 meses internadas com pneumonia grave em um hospital do Nordeste brasileiro.

Métodos. Estudo observacional, transversal, descritivo, com componente analítico. Foram avaliados 154 pacientes hospitalizados com pneumonia grave, com ou sem comprometimento pleural. O comprometimento pleural foi definido segundo achados radiológicos. As seguintes variáveis sócio-ambientais foram analisadas: faixa etária, sexo, local de residência, condições do domicilio, freqüência à creche, fumo passivo, renda familiar, presença de bens de consumo, escolaridade e trabalho extra-domiciliar da mãe ou responsável pela criança. As informações foram obtidas através de entrevistas com o responsável pelo paciente ou consulta ao prontuário médico durante a hospitalização.

Resultados. A freqüência de comprometimento pleural foi de 25,3\%. Os seguintes fatores foram associados à ocorrência de comprometimento pleural: residência em zona rural, dois cômodos ou menos no domicílio, renda familiar mensal inferior a 170 dólares e peso de nascimento $<2500 \mathrm{~g}$.

Conclusões. Os achados sugerem a necessidade de priorizar a melhoria das condições socioeconômicas e de moradia da população mais carente, principalmente aquela oriunda do meio rural. O setor saúde deve enfatizar a atenção primária, com enfoque preventivo desde o período pré-natal.

Palavras-chave Derrame pleural, inflamação pulmonar, pulmão.

As infecções respiratórias agudas (IRA) representam um dos principais problemas de saúde pública em crianças menores de 5 anos em todo o

\footnotetext{
Instituto Materno Infantil de Pernambuco (IMIP), Recife (PE), Brasil. Correspondência e pedidos de separatas devem ser enviados a Karla Danielle B. P. Costa Pinto no seguinte endereço: Rua Ricardo Salazar, 336/apto. 903, Madalena, CEP 50720-120, Recife, PE, Brasil. Fone: +55-81-3228-5529; e-mail: kardani13@hotmail.com

2 IMIP e Universidade de Pernambuco, Recife (PE), Brasil.
}

mundo (1). Enquanto nos países desenvolvidos as IRA têm importante participação na morbidade nesse grupo etário, nos países em desenvolvimento essas infecções tendem a ser mais graves e operam com componente considerável tanto na morbidade quanto na mortalidade, concentrando cerca de 20 a $40 \%$ das consultas em serviços de pediatria, 12 a $35 \%$ das internações hospitalares (2) e 19\% das mortes (3). Anualmente, cerca de 150000 crianças menores de 5 anos morrem no continente americano devido à pneumonia, que representa de 80 a $90 \%$ do total de mortes por IRA (4).

A ocorrência de complicações nos quadros de pneumonia é um dos principais determinantes do agravamento e do risco de morte nas crianças menores de 5 anos (5-8). O envolvimento da pleura nos quadros pneumônicos, se não identificado corretamente, contribui substancialmente para o agrava- 
mento do quadro clínico e para o comprometimento da função e mecânica respiratórias, determinando um maior risco de óbito por insuficiência e falência respiratórias (9-15). Nos países desenvolvidos, após o advento das sulfas e dos antibióticos, a ocorrência de derrames pleurais na população geral teve importante declínio, sendo atualmente considerada como complicação rara das pneumonias $(16,17)$. Em contrapartida, nos países em desenvolvimento, são registradas altas taxas de mortalidade por pneumonias complicadas. A Organização Pan-Americana da Saúde (OPAS) e a Organização Mundial da Saúde (OMS) (18) consideram o derrame pleural a complicação mais freqüente das pneumonias graves em crianças menores de 5 anos.

Para a realização do presente estudo foi estruturado um modelo explicativo para as possíveis associações entre comprometimento pleural na pneumonia grave em crianças menores de 5 anos e alguns fatores ligados às condições sócio-ambientais, ao hospedeiro e ao agente patogênico. Partimos do pressuposto de que os fatores determinantes do adoecimento por pneumonia grave são os mesmos responsáveis pelo comprometimento pleural, e do pressuposto de que os fatores sócio-ambientais, os fatores ligados ao hospedeiro e os ligados ao agente patogênico compartilham um mesmo nível hierárquico no determinismo da sucessão de eventos que culminam com o adoecimento da criança menor de 5 anos por pneumonia grave com posterior desenvolvimento de comprometimento pleural.

Assim, o presente trabalho teve como objetivo o estudo de associações entre determinados fatores sócio-ambientais e o desenvolvimento de comprometimento pleural na pneumonia grave em crianças menores de 5 anos tratadas em um hospital terciário de referência na Região Nordeste do Brasil.

\section{MATERIAIS E MÉTODOS}

Cerca de 6000 crianças são internadas anualmente no setor de pediatria do Instituto Materno Infantil de Pernambuco (IMIP), e aproximadamente
15\% das internações se devem a pneumonias. O IMIP é um hospital de referência no Nordeste do Brasil, que presta atendimento a todo o Estado de Pernambuco e a estados vizinhos. O presente trabalho contou com a aprovação do Comitê de Ética em Pesquisa do IMIP.

Este estudo foi observacional, transversal e descritivo, com componente analítico. Foi analisada a exposição a determinados fatores epidemiológicos (descritos mais adiante) por pacientes de 3 a 59 meses hospitalizados com pneumonia grave com ou sem comprometimento pleural $(\mathrm{CP})$, estando o $\mathrm{CP}$ presente na internação ou tendo sido desenvolvido durante o período de hospitalização no IMIP.

CP foi definido como líquido no espaço pleural ou processo inflamatório da pleura sem derrame considerandose os achados do raio $X$ de tórax, que incluem desde pequena separação pleural e opacificação dos ângulos costo-frênicos até opacificação do hemitórax e deslocamento do mediastino para o lado contra-lateral (19). Os pacientes foram inicialmente classificados de acordo com a presença ou não de $\mathrm{CP}$, procedendo-se então à análise de prevalência de variáveis epidemiológicas relacionadas a algumas condições sócio-ambientais. A amostra foi composta por 154 crianças cujas informações foram coletadas no período de 11 de julho de 2000 a 8 de maio de 2001.

Foram incluídas crianças com pneumonias graves, de acordo com os critérios da OMS (2), também adotados pelo Ministério da Saúde do Brasil (20). No momento da internação, as crianças incluídas no projeto iniciaram tratamento com ampicilina ou penicilina cristalina, conforme protocolo da pesquisa. Os critérios de exclusão incluíram: asma, doenças crônicas, doenças infecto-contagiosas, imunodeficiências, sepse e utilização de outros esquemas antibióticos no momento da admissão. Foram solicitados, no momento da internação: raio $X$ de tórax, hemograma com hemossedimentação e hemocultura.

As seguintes características sócioambientais foram estudadas: faixa etá- ria, sexo da criança, local de residência, condições do domicílio (número de cômodos, número de pessoas residentes, número de pessoas no dormitório da criança, número de crianças menores de 5 anos no domicílio), freqüência à creche, fumo passivo, renda familiar, presença de bens de consumo, escolaridade e trabalho extradomiciliar da mãe ou responsável pela criança. As informações foram obtidas mediante entrevista com a mãe ou responsável pela criança, após assinatura de consentimento livre e esclarecido.

Foi utilizado para a análise o programa Epi Info versão 6.04b (21). Os dados foram submetidos a análise bivariada, utilizando-se tabelas tipo $2 \times 2$. Os testes de associação utilizados foram o qui-quadrado $\left(\chi^{2}\right)$ de Pearson e o teste exato de Fisher (bicaudal), quando necessário. Foi utilizado o teste do $\chi^{2}$ de tendência para algumas variáveis independentes quantitativas, que foram ordenadas a fim de testar o efeito tipo dose-resposta em relação ao desfecho. Foi adotado um nível de significância estatística de 5\%, com poder de $80 \%$. A intensidade da associação foi determinada calculando-se a razão de prevalência (RP) com seus respectivos intervalos de confiança de $95 \%$ (IC95\%). O nível de referência padronizado $(R P=1)$ significou a melhor situação encontrada, ou seja, risco nulo.

\section{RESULTADOS}

Durante o período da coleta de dados, de 11 de julho de 2000 a 8 de maio de 2001, foram admitidos 350 pacientes com diagnóstico de pneumonia grave, com idade entre 3 e 59 meses. Destes, 196 não foram incluídos em função dos critérios de exclusão citados. Dos 196 pacientes excluídos, 45 apresentavam CP (23\%).

Dentre as 154 crianças incluídas no estudo, CP esteve presente em 39 , representando uma freqüência de $25,3 \%$. A média de idade das 154 crianças foi de 22,46 meses, com desvio padrão (DP) de 13,84 meses. Houve predomínio de crianças na faixa etária de menos de 24 meses. Nenhum paciente com menos de 6 meses de idade de- 
senvolveu $\mathrm{CP}$. O número de meninos foi maior $(56 \%)$ do que o número de meninas na amostra.

Não houve associação estatisticamente significativa entre a faixa etária (menores de 1 ano e de 1 a 4 anos) e o $\mathrm{CP}(P=0,57)$. Também não foi observada associação estatística entre o sexo e o CP $(P=0,11)$. Não foi possível obter informação sobre o tempo de adoecimento para um paciente. Assim, a média do tempo de adoecimento antes da internação em 153 crianças foi de 5,3 dias, com DP de 3,3 dias. A análise categorizada da variável tempo de adoecimento não evidenciou associação significativa com o CP (tabela 1).

A tabela 2 resume as informações acerca das variáveis sócio-ambientais que foram significativamente associadas a CP no presente estudo. As crianças residentes em zona rural apresentaram um risco duas vezes maior de desenvolver CP do que as crianças residentes em área urbana $(P=0,01)$. Da mesma forma, o risco de CP foi duas vezes maior para as crianças que moravam em domicílios com até dois cômodos em relação às crianças cujos domicílios possuíam três ou mais cômodos $(P=0,02)$. Além disso, houve associação significativa entre a renda familiar mensal e o CP $(P=0,03)$. As

TABELA 1. Distribuição do comprometimento pleural segundo o tempo de adoecimento antes da internação em crianças menores de 5 anos com pneumonia grave, Recife (PE), Brasil, julho de 2000 a maio de 2001

\begin{tabular}{|c|c|c|c|c|c|c|}
\hline \multirow{3}{*}{$\begin{array}{c}\text { Tempo de } \\
\text { adoecimento (dias) }\end{array}$} & \multicolumn{4}{|c|}{ Comprometimento pleural } & \multirow{3}{*}{$\begin{array}{l}\text { Razão de prevalência } \\
\text { (IC95\%) }\end{array}$} & \multirow[b]{3}{*}{$P\left(\chi^{2}\right)$} \\
\hline & \multicolumn{2}{|c|}{ Sim } & \multicolumn{2}{|c|}{ Não } & & \\
\hline & No. & $\%$ & No. & $\%$ & & \\
\hline Até 7 & 32 & 24,2 & 100 & 75,8 & $1,18(0,56-2,47)$ & 0,10 \\
\hline$\geq 8$ & 6 & 28,6 & 15 & 71,4 & & 0,66 \\
\hline Total & 38 & 24,8 & 115 & 75,2 & & \\
\hline
\end{tabular}

crianças cujas famílias dispunham de renda mensal inferior a 170 dólares apresentaram um risco duas vezes maior para CP. Também foi observada uma associação significativa entre a variável peso de nascimento e o $\mathrm{CP}(P=$ 0,$05 ; \mathrm{RP}=1,85 ; \mathrm{IC} 95 \%=1,03$ a 3,34).

A análise das seguintes características relacionadas ao confinamento não evidenciou associação com o CP: número de residentes $(P=0,16)$, número de pessoas no quarto de dormir da criança $(P=0,20)$, número de crianças menores de 5 anos $(P=0,16)$ e freqüência à creche $(P=1,00)$. Também não foi observada associação estatisticamente significativa entre o $\mathrm{CP}$ e o fumo passivo $(P=0,64)$, bens de con- sumo, tais como TV $(P=0,29)$, rádio $(P=0,56)$, geladeira $(P=0,21)$ e carro $(P=0,07)$, escolaridade $(P=0,24)$ e trabalho extra-domiciliar da mãe ou responsável pela criança $(P=0,17)$.

\section{DISCUSSÃO}

Este estudo teve por objetivo identificar possíveis fatores de risco sócioambientais associados ao desenvolvimento de $\mathrm{CP}$ em crianças com idade entre 3 e 59 meses hospitalizadas com pneumonia grave no IMIP no período de 11 de julho de 2000 a 8 de maio de 2001. O estudo tem algumas limitações quanto à sua validade externa, visto

TABELA 2. Distribuição do comprometimento pleural segundo variáveis sócio-ambientais em crianças menores de 5 anos com pneumonia grave, Recife (PE), Brasil, julho de 2000 a maio de 2001

\begin{tabular}{|c|c|c|c|c|c|c|c|}
\hline \multirow[b]{3}{*}{ Variável } & \multicolumn{5}{|c|}{ Comprometimento pleural } & \multirow[b]{3}{*}{ Razão de prevalência (IC95\%) } & \multirow[b]{3}{*}{$P\left(\chi^{2}\right)$} \\
\hline & \multicolumn{2}{|c|}{ Sim } & \multicolumn{2}{|c|}{ Não } & \multirow[b]{2}{*}{ Total } & & \\
\hline & No. & $\%$ & No. & $\%$ & & & \\
\hline \multicolumn{8}{|c|}{ Local de residência } \\
\hline Zona rural & 12 & 44,4 & 15 & 55,6 & 27 & \multirow[t]{3}{*}{$2,09(1,22$ a 3,58) } & 6,33 \\
\hline Zona urbana & 27 & 21,3 & 100 & 78,7 & 127 & & 0,01 \\
\hline Total & 39 & 25,3 & 115 & 74,7 & 154 & & \\
\hline \multicolumn{8}{|c|}{ Número de cômodos } \\
\hline Até dois & 11 & 44,0 & 14 & 56,0 & 25 & \multirow[t]{3}{*}{$2,03(1,17-3,52)$} & 5,50 \\
\hline Três ou mais & 28 & 21,7 & 101 & 78,3 & 129 & & 0,02 \\
\hline Total & 39 & 25,3 & 115 & 74,7 & 154 & & \\
\hline \multicolumn{8}{|c|}{ Renda familiar (dólares) } \\
\hline$\leq 170$ & 30 & 31,9 & 64 & 68,1 & 94 & \multirow[t]{3}{*}{$1,99(1,02-3,87)$} & 4,58 \\
\hline$\geq 171$ & 9 & 16,1 & 47 & 83,9 & 56 & & 0,03 \\
\hline Total & 39 & 26,0 & 111 & 74,0 & 150 & & \\
\hline \multicolumn{8}{|c|}{ Peso de nascimento (g) } \\
\hline$<2500$ & 9 & 42,9 & 12 & 57,1 & 21 & \multirow[t]{3}{*}{$1,85(1,03-3,34)$} & 3,61 \\
\hline$\geq 2500$ & 28 & 23,1 & 93 & 76,9 & 121 & & 0,05 \\
\hline Total & 37 & 26,1 & 105 & 73,9 & 142 & & \\
\hline
\end{tabular}


que a amostra se restringiu às crianças internadas com pneumonia grave no IMIP. Outra questão a ser considerada é o viés de prevalência de $\mathrm{CP}$ relacionado às crianças que foram excluídas; no entanto, a freqüência de CP observada nos 196 pacientes excluídos foi de $23 \%$, portanto muito próxima da freqüência obtida para a amostra estudada $(25,3 \%)$. A maior parte das crianças (149) já apresentava CP no momento da internação.

As variáveis potencialmente confundidoras, como idade e sexo, não foram controladas neste estudo, o que pode dificultar a interpretação de alguns resultados. Quanto à possibilidade de problemas relativos à classificação da presença ou ausência de CP, acreditamos que esses tenham sido eliminados mediante a utilização de critérios radiológicos para o desfecho e o adequado seguimento da criança durante a internação, no intuito de se detectar qualquer sinal que pudesse sugerir $\mathrm{CP}$ durante o tratamento do paciente. A equipe de coleta de dados foi composta por médicos pediatras, funcionários do IMIP, que dispunham de um serviço de radiologia qualificado para esclarecimento de qualquer caso duvidoso em relação à presença ou não de CP. Assim, a análise das radiografias de tórax merece credibilidade.

A idade tem sido implicada como fator de risco para agravamento e mortalidade nas IRA graves, principalmente em crianças menores de 1 ano $(2,22,23)$. Não foram observadas na amostra crianças abaixo de 6 meses de idade que desenvolvessem CP. Isso pode sugerir a existência de um componente imunitário, por exemplo, a presença de anticorpos transplacentários como fator de proteção. No presente estudo, a faixa etária categorizada das crianças não constituiu fator de risco para o desenvolvimento de CP. Uma possível explicação para este fato pode ser o tamanho da amostra, que pode ter sido insuficiente para detectar essa diferença na análise bivariada.

Quanto à variável sexo, outros estudos de fatores de risco para IRA também relatam um maior número de casos em pacientes do sexo masculino (24-26). Todavia, o sexo não representou risco após análise dos resultados desses trabalhos, da mesma forma como observado no presente estudo.

A demora no início do tratamento antibiótico vem sendo implicada na literatura como um fator estritamente relacionado ao aparecimento de derrame pleural (18). Neste estudo, a análise da variável tempo de adoecimento antes da internação não evidenciou associação estatisticamente significativa com CP. Seria necessário um novo estudo para avaliar o valor da antibioticoterapia iniciada antes da internação em termos de uma possível associação com proteção contra o desenvolvimento de derrame pleural.

Residir em zona rural representou um risco duas vezes maior para $\mathrm{CP}$ em comparação com residir em zona urbana. Alguns fatores podem estar interferindo na interpretação desse resultado, tais como a dificuldade de acesso aos serviços de saúde, as condições socioeconômicas e o clima da região. $\mathrm{O}$ meio rural do Estado de Pernambuco apresenta importantes deficiências referentes a saneamento básico, emprego, renda, educação, nutrição e assistência à saúde (27). Essas características, relacionadas às questões socioeconômicas e nutricionais, podem reforçar a hipótese de associação de algumas dessas variáveis com um modelo explicativo para agravamento das pneumonias graves e desenvolvimento de CP.

Dentre as variáveis relacionadas ao confinamento, apenas a relativa ao número de cômodos do domicílio apresentou associação estatística com o desenvolvimento de CP. Há de se considerar que essa variável, dentre as demais apresentadas, é uma das mais diretamente ligadas às condições socioeconômicas da família. Por sua vez, os valores de significância estatística encontrados na análise da variável fumo passivo indicam que, para se inferir a possibilidade da existência de alguma diferença entre os dois grupos, seria necessário um tamanho de amostra bastante superior ao estudado na análise do presente estudo.
Dentre as crianças estudadas, 2,6\% não dispunham de informação sobre a renda familiar mensal. Um percentual de $62,25 \%$ das famílias informou renda igual ou inferior a US\$170, representando um risco duas vezes maior para desenvolvimento de $\mathrm{CP}$, em comparação com as famílias com renda familiar superior a esse valor. Tal achado pode reforçar a hipótese de forte influência dos fatores econômicos na associação com o agravamento das pneumonias e o desenvolvimento de CP. Quanto à escolaridade da mãe ou responsável pela criança, o fato de existirem apenas 16 casos de analfabetismo pode ter prejudicado a análise dessa variável. Também não foi observada associação estatística entre o desenvolvimento de $\mathrm{CP}$ e o trabalho extra-domiciliar da mãe ou responsável pela criança. Um percentual de $73 \%$ das mães ou responsáveis não trabalhavam fora do domicílio. Em relação à análise da variável peso de nascimento, observamos uma chance $85 \%$ maior de desenvolver $\mathrm{CP}$ entre as crianças com peso ao nascer inferior a $2500 \mathrm{~g} \mathrm{em}$ comparação às crianças que nasceram com peso igual ou superior a $2500 \mathrm{~g}$.

Do ponto de vista intervencionista, os fatores sócio-ambientais apresentados demonstram a clara dependência do processo de saúde e doença quanto à necessidade de organização de outros setores estritamente relacionados à qualidade de vida da população. As medidas de intervenção que poderiam ser aventadas para diminuir a freqüência de $C P$ na população de crianças atendidas com pneumonia grave deveriam ser direcionadas primordialmente à melhoria das condições socioeconômicas e de moradia da população mais carente, principalmente aquela oriunda do meio rural. É fundamental impedir o adoecimento e o possível agravamento dos quadros de pneumonia em crianças menores de 5 anos. $\mathrm{O}$ setor saúde pode contribuir nesse sentido se abordar de forma integral os principais problemas de saúde prevalentes na infância, com ênfase na atenção primária e com enfoque preventivo desde o período pré-natal. 


\section{REFERÊNCIAS}

1. Benguigui Y. Alguns resultados de experiências com o controle de infecções respiratórias agudas (IRA). Em: Benguigui $Y$, ed. Investigações operacionais sobre o controle das infecções respiratórias agudas (IRA). Washington, D.C.: Organização Pan-Americana da Saúde; 1997. Pp. 159-163.

2. Bulla A, Hitze KL. Acute respiratory infections: a review. Bull World Health Organ. 1978;56(3):481-498.

3. World Health Organization. Integrated Management of Childhood Illness (IMCI). Disponível em: http://www.who.int/childadolescent-health/integr.htm. Acessado em dezembro de 2003.

4. Benguigui $Y$, Land S, Paganini JM, Yunes J, eds. Ações de saúde materno-infantil a nível local segundo as metas da Cúpula Mundial em Favor da Infância. Washington: Organização Pan-Americana da Saúde/Organização Mundial da Saúde; 1997. (Série HCT/AIEPI4).

5. Freij BJ, Kusmiesz H, Nelson JD, MacCracken GH. Parapneumonic effusions and empyema in hospitalized children: a retrospective review of 227 cases. Pediatr Infect Dis J. 1984; 3(6):578-591.

6. Sánchez JO, Martínez AS. Infección pleuropulmonar y sus complicaciones en el niño. Tratamiento quirúrgico. Experiencia de 20 años. Cir Ciruj. 1995;63(4):130-136.

7. Victora CG. Fatores de risco nas IRA baixas. Em: Benguigui $Y$, Antuñano FJL, Schmunis G, Yunes J, eds. Infecções respiratórias em crianças. Washington, D.C.: Organização PanAmericana da Saúde/Organização Mundial da Saúde; 1998. Pp. 43-61. (Série HCT/AIEPI1.P).

8. Berezin EN, Kiertzman B, Brandileone MC, Masson L, Toda EN, Lopes CR, et al. Complicated parapneumonic effusion caused by Streptococcus pneumoniae $(\mathrm{Sp})$ in hospitalized children in Brazil: clinical and microbiologic characteristics. Em: 39th Interscience Conference on Antimicrobial Agents and Chemotherapy (ICAAC); 1999 Set 26-29; San Francisco, California. (Abstract 1064).

9. Escobar MM, Rubio LM, Bravo SE. Derrame pleural parapneumónico y empiema en niños: Hospital General de Medellín. Revista de la Facultad de Medicina del Instituto de Ciencias de la Salud CES (Medellín). 1989;3(1): 15-18.
10. Rodrigues JR, Rozov T, Melles CEA, Brandileone MCC, Bocardin NB, Okay Y. Derrames pleurais parapneumônicos na infância: análise da importância dos métodos laboratoriais no diagnóstico etiológico. Em: Benguigui $Y$, ed. Investigações operacionais sobre o controle das infecções respiratórias agudas (IRA). Washington, D.C.: Organização Pan-Americana da Saúde; 1997. Pp. 143-155.

11. Freitas AEH, Gomes VC. Derrames pleurais parapneumônicos em crianças: estudo de 77 pacientes internados no Hospital Infantil Albert Sabin, Fortaleza, Ceará. Pediatr Atual. 1998;11(11):9-13.

12. Carballal G, Siminovich M, Mutach $P$ Cerqueiro MC, Ávila M, Salomón H, et al. Etiological, clinical and pathological analysis of 31 fatal cases of acute respiratory tract infection in Argentinean children under 5 years of age. Rev Infect Dis. 1990;12(Suppl 8):1074S$1080 \mathrm{~S}$.

13. Munglani R, Kennedy J. Paediatric parapneumonic effusions: a review of 16 cases. Respir Med. 1991;85(2):117-119.

14. Schutze GE, Jacobs RF. Management of community-acquired bacterial pneumonia in hospitalized children. Pediatr Infect Dis J. 1992;11(2):160-164.

15. Sant'anna CC. Condutas padronizadas em pneumonias agudas na infância. Pediatr Atual. 1999;12(3):50-58.

16. Ravitch MM, Fein $R$. The changing picture of pneumonia and empyema in infants and children. JAMA. 1961;175:1039-1044.

17. Hardie WD, Roberts NE, Reising SF, Christie CDC. Complicated parapneumonic effusions in children caused by penicillin-nonsusceptible Streptococcus pneumoniae. Pediatrics. 1998; 101(3 Pt 1):388-392.

18. Benguigui Y, Bossio JC, Fernández HR. Investigaciones operativas sobre Atención Integrada a las Enfermedades Prevalentes de la Infancia (AIEPI). Washington, D.C.: Organización Panamericana de la Salud/Organización Mundial de la Salud; 2001. (Serie HCT/ AIEPI-27.E)

19. Ruvinski R, Balanzal M. Pneumonias bacterianas e virais. Em: Benguigui $Y$, Antuñano FJL, Schmunis G, Yunes J, eds. Infecções respiratórias em crianças. Washington, D.C.: Organização Pan-Americana da Saúde/Organização Mundial da Saúde; 1998. Pp. 217-251. (Série HCT/AIEPI-1.P)
20. Brasil, Ministério da Saúde. Manejo de infecções respiratórias agudas em crianças: avaliação em unidades de saúde de seis capitais do Brasil. Brasília; 2000.

21. Dean JG, Dean JA, Coulombier D, Brendel KA, Smith DC, Burton AH, et al. Epi Info 6.0: a word processing database and statistics program for epidemiology on IBM compatible computers. Atlanta, Georgia: Centers for Disease Control and Prevention; 1994.

22. Benguigui Y. Controle das infecções respiratórias agudas em crianças, Pará, Brasil. Bol Of Sanit Panam. 1987;102(1):36-48.

23. Demers AM, Morency P, Mberyoyaah F, Jaffar S, Blais C, Somsé P, et al. Risk factors for mortality among children hospitalized because of acute respiratory infections in Bangui, Central African Republic. Pediatr Infect Dis J. 2000;19(5):424-432.

24. Amaral J, Menezes AMB, Halpern R, Victora CG, Barros FC. Prevalência e fatores de risco para infecção respiratória aguda em crianças aos seis meses de vida em Pelotas, RS. Em: Benguigui $Y$, ed. Investigações operacionais sobre o controle das infecções respiratórias agudas (IRA). Washington: Organização PanAmericana da Saúde; 1997. Pp. 85-97.

25. Banajeh SM. Outcome for children under 5 years hospitalized with severe acute lower respiratory tract infections in Yemen: a 5 year experience. J Trop Pediatr. 1998;44(6):343-346.

26. Broor S, Pandey RM, Ghosh M, Maitrey RS, Lodha R, Singhal T, et al. Risk factors for severe acute lower respiratory tract infection in under-five children. Indian Pediatr. 2001; 38(12):1361-1369.

27. Brasil, Ministério da Saúde. II Pesquisa Estadual de Saúde e Nutrição: saúde, nutrição, alimentação e condições socioeconômicas do estado de Pernambuco. Recife: Secretaria de Saúde/Instituto Nacional de Alimentação e Nutrição/Instituto Materno Infantil de Pernambuco/Universidade Federal de Pernambuco, Departamento de Nutrição; 1998.

Manuscrito recebido em 6 de dezembro de 2002. Aceito em versão revisada el 7 de setembro de 2003 
ABSTRACT Objective. To determine social and environmental risk factors associated with pleural involvement among children from 3 to 59 months old who were hospitalized with severe pneumonia in northeastern Brazil.

\section{Analysis of social and environmental risk for pleural involvement in severe pneumonia in children younger than 5 years of age}

Methods. This was an observational and descriptive cross-sectional study with an analytical component. We evaluated 154 patients hospitalized due to severe pneumonia, with or without pleural involvement. Pleural involvement was determined based on radiological findings. The following variables were analyzed: age, sex, place of residence, housing conditions, day care attendance, passive smoking, family income, presence of consumer goods in the home (e.g., television, radio, refrigerator, automobile), and schooling and occupation of the mother or other caregiver. The information was obtained by interviewing the mother or other caregiver or by consulting the inpatient medical chart.

Results. The frequency of pleural involvement among the 154 patients evaluated was $25.3 \%$. The following factors were associated with pleural involvement: living in a rural area, being in a household with two or fewer rooms, family income below 170 U.S. dollars per month, and birthweight $<2500 \mathrm{~g}$.

Conclusions. These findings indicate the need to improve the socioeconomic and living conditions of the less-privileged population, especially in rural areas. The health sector should emphasize primary care, focusing on a preventive approach beginning in the prenatal period.

\title{
Nominations Sought for 2004 Abraham Horwitz Award for Leadership in Inter-American Health
}

Nominations are being accepted for the 2004 Abraham Horwitz Award for Leadership in Inter-American Health. The Horwitz Award is granted each year by the Pan American Health and Education Foundation (PAHEF), which is a nonprofit foundation and a partner of the Pan American Health Organization (PAHO).

Created in 1975, the Horwitz Award recognizes public health leaders whose commitment to public health has stimulated excellence among their peers, staff, and other public health professionals and has impacted the health of populations in the Region of the Americas. Nominees may be active in their careers, be active though in formal retirement, or be retired after having had an outstanding lifetime career. In addition to recognizing the honoree's professional accomplishments, the Horwitz Award calls attention to health concerns in the Americas.

A select, international jury of public health professionals reviews the nominations and then recommends a candidate to the PAHEF Board of Trustees for their final selection. The winner receives US $\$ 5000$, a certificate of honor, and a paid trip to Washington, D.C., to address the ministers of health from the Region of the Americas and other persons attending the Annual Meeting of PAHO.

Nominations must be made by submitting a letter of introduction that is no longer than one page, along with a completed Horwitz Award nomination form; the nomination form is available from PAHEF. The letter and the completed form must be in English. Nominations must be received no later than 30 May 2004.

\author{
Information: \\ Pan American Health and Education Foundation \\ 525 23rd Street, N.W. \\ Washington, D.C. 20037 \\ United States of America \\ Telephone: 202-974-3416 \\ Fax: 202-974-3636 \\ e-mail: foundation@paho.org
}

Internet (English): http://www.paho.org/English/PAHEF/horwitz.htm

Internet (Spanish): http://www.paho.org/Spanish/PAHEF/horwitz.htm 